\title{
OFERTA DOS CURSOS DE GRADUAÇÃO EM ENFERMAGEM NO ESTADO DE MINAS GERAIS ${ }^{1}$
}

\author{
Kênia Lara Silva², Roseni Rosangela de Sena ${ }^{3}$, Tatiana Silva Tavares ${ }^{4}$, Ana Carolina Silva Martins ${ }^{5}$
}

\footnotetext{
${ }^{1}$ Pesquisa financiada pela Fundação de Amparo à Pesquisa do Estado de Minas Gerais (FAPEMIG)

${ }^{2}$ Doutora em Enfermagem. Professora Adjunto do Departamento de Enfermagem Aplicada da Escola de Enfermagem da Universidade Federal de Minas Gerais (UFMG). Minas Gerais, Brasil. E-mail: kenialara17@yahoo.com.br

${ }^{3}$ Doutora em Enfermagem. Professora Emérita da Escola de Enfermagem da UFMG. Bolsista de Produtividade CNPq. Minas Gerais, Brasil. E-mail: rosenisena@uol.com.br

${ }^{4}$ Enfermeira. Discente do curso de Mestrado da Escola de Enfermagem da UFMG. Bolsista FAPEMIG. Minas Gerais, Brasil. E-mail: tatianasilvatavares@yahoo.com.br

${ }^{5}$ Acadêmica do curso de Graduação em Enfermagem da Escola de Enfermagem da UFMG. Bolsista PIBIC/CNPq. Minas Gerais, Brasil. E-mail: anacarolinasilvamartins@yahoo.com.br
}

RESUMO: O objetivo do estudo foi analisar oferta de cursos de enfermagem no Estado de Minas Gerais, estabelecendo relações com as políticas públicas de educação no país. Estudo descritivo transversal, de abordagem quantitativa, teve como fonte o banco de dados e-MEC do Ministério da Educação e Cultura, consultado em fevereiro de 2010. Utilizou-se o universo dos cursos de graduação em enfermagem. Os achados revelam a existência de 126 cursos no estado na modalidade presencial que apresentaram expansão a partir do ano 2000 com concentração nas regiões mais desenvolvidas do estado. O aumento do número de cursos se deu predominantemente nas instituições de natureza privada e no turno da noite. A expansão dos cursos de enfermagem está relacionada às políticas de educação. A autonomia pedagógica, administrativa e de gestão financeira das instituições de ensino favorecem o aumento do número de instituições. DESCRITORES: Educação em enfermagem. Ensino superior. Instituições de ensino superior. Políticas públicas.

\section{OFFER OF UNDERGRADUATE NURSING PROGRAMS IN MINAS GERAIS}

\begin{abstract}
The aim of this study is to analyze the distribution of undergraduate nursing programs in the state of Minas Gerais, Brazil, and make associations between them and public education policy. It is a descriptive cross-sectional study whose source was the e-MEC database from the Brazilian Ministry of Culture and Education, accessed in February of 2010, using the universe of undergraduate nursing programs. Data indicates that there are 126 courses with the traditional in-class modality which have presented expansion since 2000, concentrating in the state's more developed regions. Course offerings have increased mainly in private institutions and in night shifts. The expansion of nursing courses is related to public education policy. Educational, administrative, and the financial management autonomy of educational institutions favor the increased number of institutions.
\end{abstract}

DESCRIPTORS: Education, nursing. Education, higher. Higher education institutions. Public Policies.

\section{OFERTA DE CURSOS DE ENFERMERÍA EN EL ESTADO DE MINAS GERAIS}

RESUMEN: Este estudio tuvo como objetivo analizar la distribución de la oferta de cursos de enfermería en el Estado de Minas Gerais y establecer los vínculos con las políticas públicas de educación. Estudio descriptivo y transversal cuya fuente fue la base de datos e-MEC del Ministerio de Educación y Cultura, consultada en febrero de 2010. Se utilizó el universo de los cursos de enfermería. Los datos indican que hay 126 cursos en el Estado en la modalidad presencial, se expandieron a partir del 2.000 y se concentran en las regiones más desarrolladas del Estado. El aumento del número de cursos se produjo sobre todo en las instituciones privadas y en los turnos nocturnos. La expansión de los cursos de enfermería está relacionada con las políticas públicas de educación. La autonomía pedagógica, administrativa y de gestión financiera de los institutos de enseñanza ayudan a aumentar el número de instituciones.

DESCRIPTORES: Educación en enfermería. Educación superior. Instituciones de enseñanza superior. Políticas públicas. 


\section{INTRODUÇÃO}

A trajetória dos cursos graduação em enfermagem no Brasil é marcada pela influência das transformações no quadro político, econômico, social da educação e da saúde no país e no mundo, o que tem sido determinante das características do ensino e do perfil dos enfermeiros. As necessidades do mercado de trabalho, reflexo das políticas econômicas, têm influenciado a criação de escolas e a orientação da formação no Brasil. ${ }^{1}$ Desta forma, podemos afirmar que, por mais que a formação em saúde esteja direcionada para atender às orientações do sistema público de saúde, elas não escapam às tendências racionalizadoras, orientadas ao mercado e à competição.

O marco do ensino formal de enfermagem se dá em 1890 com a criação da Escola Profissional de Enfermeiros no Hospício Nacional de Alienados com o propósito de preparar profissionais para os hospícios e hospitais civis e militares. Em 1901 foi criado o Hospital Evangélico, destinado a atender os estrangeiros residentes no Brasil. Por volta de 1916, a Cruz Vermelha brasileira, devido à Primeira Guerra Mundial, deu início a um curso de enfermagem, na cidade do Rio de Janeiro, para preparar voluntários para as emergências de guerra. ${ }^{2}$

No início da década de 1920, foi criada a atual Escola de Enfermagem Anna Nery, considerada a primeira escola de enfermagem moderna do país. Na Escola, estabelecia-se a formação com cunho preventivo para responder aos problemas da saúde pública enfrentados pelo país naquele momento. ${ }^{3}$

A década de 30 é um marco para a evolução dos cursos de enfermagem no país, visto a necessidade de uma força de trabalho qualificada, em decorrência do processo de reorganização político-econômica. Na década de 1940, com o fortalecimento do processo de industrialização, o estado brasileiro cria a lei $\mathrm{n}^{\circ} 775 / 49$, que propõe a ampliação do número de escolas, tornando obrigatória a existência do ensino de enfermagem em todo centro universitário ou sede de faculdades de medicina, e define um ensino focado na área hospitalar. ${ }^{2}$ Nesta época, as recomendações do Relatório Flexner marcaram o ensino na área da saúde, com um eixo voltado para a prática da atenção individual e para o modelo curativista das doenças. ${ }^{4}$

Nas décadas de 1940 e 1950, em decorrência do desenvolvimento técnico-científico e da utilização de equipamentos modernos e sofisticados para a época, expressos na criação dos centros de excelência de tratamento intensivo, o ensino de enfermagem passa por transformações. ${ }^{3}$

Nos anos 1980, o advento da VIII Conferência Nacional de Saúde e a promulgação da Constituição em 1988 expressaram um processo de luta de diferentes atores da sociedade por aumento dos direitos sociais. ${ }^{2}$ Para dar resposta à necessidade de transformação na educação de enfermagem, a Associação Brasileira de Enfermagem (ABEn) realizou, no período de 1989 a 1992, um movimento nacional para discutir e propor um Currículo Mínimo de Enfermagem, que foi regulamentado pelo Ministério da Educação em $1994 .^{5}$

Entre os elementos considerados como de fundamental importância, para fazer cumprir o que foi idealizado no Currículo Mínimo de Enfermagem, incluiam-se a modificação do modelo acadêmico, incluindo ampliação dos cenários de ensino para os diversos níveis de serviços de saúde, educação e das organizações comunitárias; inovações dos conteúdos temáticos nos currículos de enfermagem; inovações das metodologias de ensino-aprendizagem; preparação pedagógica e didática dos docentes; e modificação e implementação de novos modelos de avaliação do processo ensino-aprendizagem. ${ }^{6}$

A implantação do Currículo Mínimo estava, ainda, em processo, quando, em 1996, foi promulgada a Lei de Diretrizes e Bases da Educação Nacional (LDB), que extinguiu os Currículos Mínimos dos cursos de graduação, e estabeleceu as diretrizes curriculares na reordenação da formação superior. $^{2,7}$

A partir de 1997, as Escolas de Enfermagem do Brasil iniciam a implantação do denominado Novo Currículo, tendo como referencial a LDB, o que exigiu uma reordenação e readequação das estruturas acadêmico-administrativas das escolas e um programa de capacitação docente para concretizar o propósito de transformação do modelo de formação do enfermeiro. ${ }^{8}$

A LDB trouxe novas responsabilidades para as instituições de ensino superior, pois permitia a formação de diferentes perfis profissionais, a partir da vocação de cada curso/escola ${ }^{1}$ e, desta forma, poderiam melhor se adaptar as necessidades regionais do mercado de trabalho.

O final do século XX é marcado pelo movimento de construção de novos paradigmas, com repercussões na organização dos serviços de saúde, nas concepções de promoção à saúde, prevenção de agravos e tratamento, fruto da conso- 
lidação do SUS. Também no modelo de formação dos profissionais de saúde houve repercussões. ${ }^{9}$

Atendendo a esse movimento, as Diretrizes Curriculares Nacionais (DCN) para os cursos de graduação na área de saúde, publicadas em 2001, passam a servir como referência para a construção dos Projetos Políticos-pedagógicos pelas escolas. A promulgação das DCN representou um marco legal, ético e político no direcionamento da formação dos profissionais enfermeiros expressando expectativas de mudanças no processo de formação em consonância com o Movimento da Reforma Sanitária brasileira e o SUS. ${ }^{10}$

A análise deste percurso histórico permite afirmar que, nos diferentes períodos, o ensino de enfermagem esteve influenciado pelas políticas de saúde e de educação, refletindo a necessidade de analisar o contexto histórico e econômico vivenciado pela nossa sociedade, como determinantes da formação de enfermeiros.

Segundo o banco de dados do Ministério da Educação e Cultura (MEC), sobre instituições de Educação Superior e Cursos Cadastrados, e-MEC*, contávamos, em fevereiro de 2010, com 598 cursos de graduação em enfermagem ativos no Brasil, em instituições públicas e privadas. Destes, 126 estão situados no estado de Minas Gerais.

Apesar do expressivo quantitativo da força de trabalho de enfermagem, em número absoluto, e da ampliação do número de escolas na última década, em todo o país, a concentração de escolas de enfermagem e a disponibilidade de enfermeiros por habitante são bastante desiguais nas diversas regiões, considerando as dimensões territoriais do Brasil, e seu contingente populacional. As escolas de enfermagem concentram-se nas regiões de maior densidade populacional e de concentração de renda do País, acompanhando a distribuição do Produto Interno Bruto (PIB), reafirmando as desigualdades regionais. ${ }^{11}$

Neste contexto, tomamos como indagação a expansão do número de escolas de enfermagem no país. Por um lado, este fenômeno pode representar democratização do acesso ao ensino superior, maior disponibilidade de profissionais no mercado, com a qualidade necessária e com aderência às demandas de saúde da população. Por outro lado, a expansão do número de cursos pode estar ocorrendo sem uma estreita relação com as políticas de saúde, em relação a qualidade e a quantidade dos profissionais requeridos para a consolidação do SUS. Ou seja, pode estar ocorrendo uma resposta à oferta de postos de trabalho sem direcionamento da formação para romper com as situações de desigualdades e iniquidades que afetam a sociedade em nosso país.

Diante disto, o objetivo deste estudo foi analisar a oferta de cursos de enfermagem no estado de Minas Gerais, discutindo a expansão e distribuição dos cursos no estado e a relação com as políticas de educação no País.

\section{MÉTODO}

Trata-se de um estudo descritivo e transversal, de abordagem quantitativa. Utilizou-se o universo de cursos de enfermagem na modalidade presencial registrados no banco de dados e-MEC, do MEC, em fevereiro de 2010.

Foram definidas como variáveis do estudo macro e microrregião de inserção de oferecimento do curso, natureza das instituições ofertantes, modalidade do curso, ano de fundação, turno de oferecimento, número de semestres para integralização do curso e carga horária total do curso. Os dados obtidos foram tabulados e submetidos a análise descritiva por meio do software Excel.

O estudo foi aprovado pelo comitê de Ética e pesquisa da Universidade Federal de Minas Gerais sob parecer ETIC 435/08.

\section{RESULTADOS}

Os achados revelam a existência de 126 cursos de enfermagem no Estado de Minas Gerais, na modalidade presencial, sendo 10 disponibilizados por instituições públicas e 116 por instituições privadas. O curso mais antigo do estado foi iniciado em 1934 em uma instituição pública federal e o segundo, em 1945, em uma instituição privada de orientação religiosa. Dessa época até 1997 , existiam 10 cursos no Estado de Minas Gerais ofertados, principalmente, em instituições públicas, com exceção de três cursos em instituições privadas.

A análise dos dados permite evidenciar que a partir do ano 2000 há uma expressiva expansão da oferta de cursos de enfermagem no Estado, com a criação de uma média de 14,6 cursos por ano, entre os anos de 2002 e 2007. A figura 1 revela o aumento significativo no número de cursos de graduação ocorrido neste período. A partir do ano de 2007, há uma tendência à diminuição do ritmo de crescimento.

* Disponível em: http://emec.mec.gov.br/ 


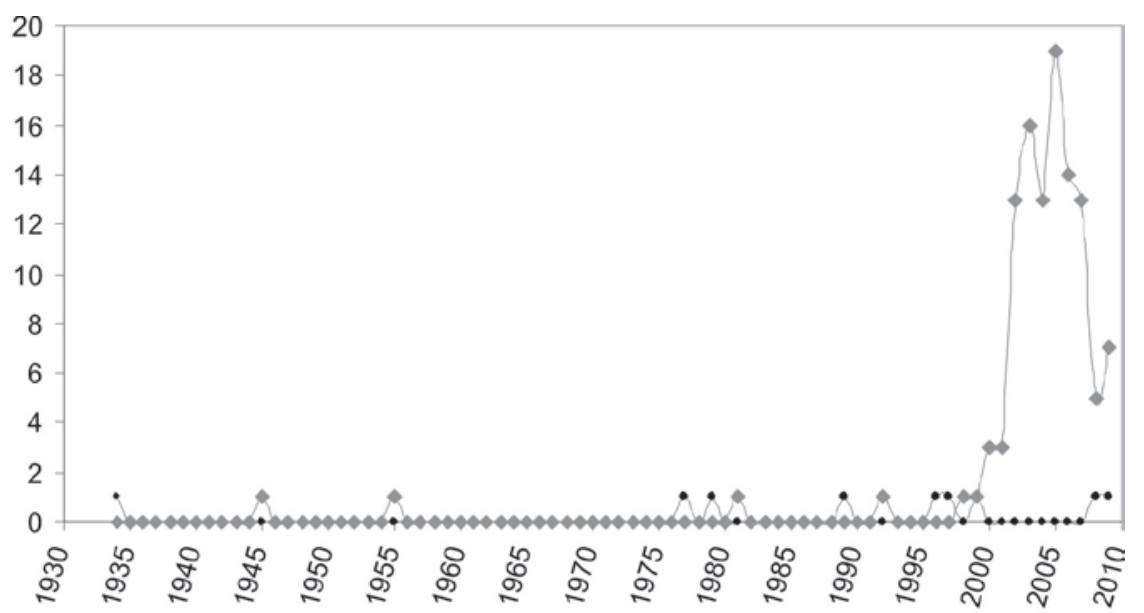

-... Cursos criados em IES pública $\longrightarrow$ Cursos criados em IES privada

Fonte: Brasil - Ministerio da Educação - 2010

Figura 1 - Número de cursos de graduação em enfermagem criados por ano de acordo com a categoria administrativa em Minas Gerais (1934-2010), Belo Horizonte, MG, 2010

Os resultados evidenciam a diferença no aumento do número de cursos nas instituições públicas e privadas. Enquanto nas instituições públicas a oferta de cursos de enfermagem manteve-se constante, nas privadas a oferta começou a aumentar no final da década de 1990 e apresentou crescimento abrupto a partir de 2000.

Os cursos de graduação em enfermagem estão distribuídos em todas as macrorregiões de Minas Gerais, com maior concentração nas macro-regiões Centro e Sul do estado, conforme demonstrado na figura 2. Contudo, há vazios nas regiões Noroeste, Nordeste, Norte e, em especial, na região do Jequitinhonha, a menos desenvolvida do Estado, onde há também ausência de cursos de graduação de enfermagem em instituições privadas. Ressalta-se a criação recente de cursos em instituições públicas com alocação nas macrorregiões Oeste e Leste do Sul do Estado, respectivamente, em 2008 e 2009.

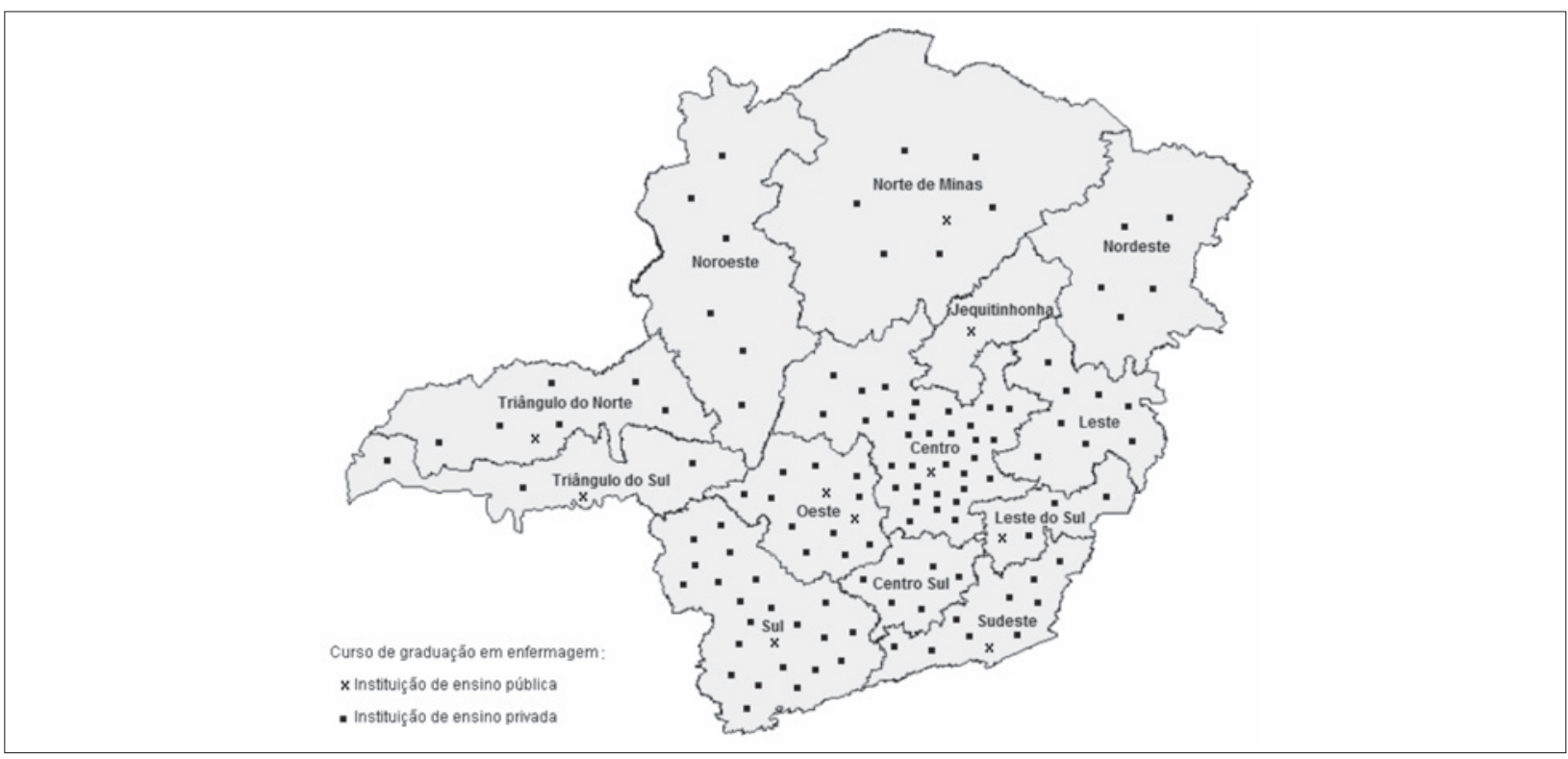

Fonte: Brasil - Ministério da Educação e Ministério da Cultura - 2010

Figura 2 - Distribuição dos cursos de graduação em enfermagem por categoria administrativa nas macro-regiões do Estado de Minas Gerais, Belo Horizonte, MG, 2010 
A figura 3 demonstra que os cursos de enfermagem do Estado são ofertados predominantemente no período diurno, embora a expansão tenha sido caracterizada pelo aumento significativo da oferta de cursos noturnos em instituições privadas.

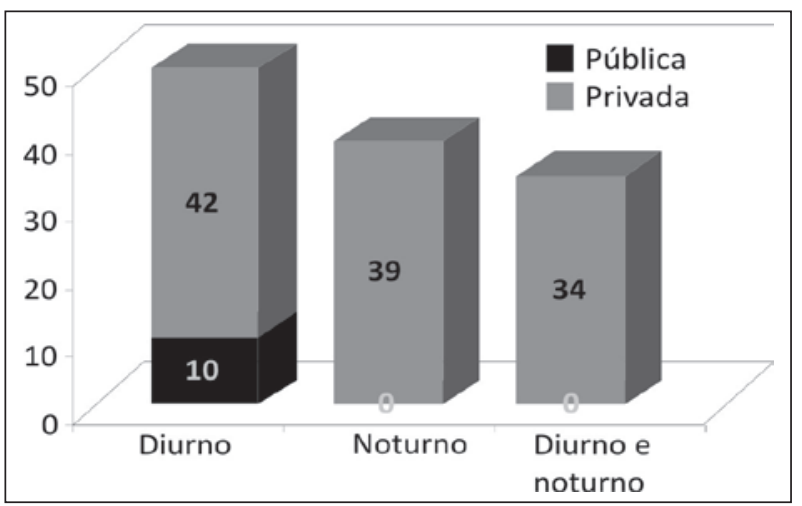

Fonte: Brasil - Ministerio da Educação - 2010

Figura 3 - Número de cursos de graduação em enfermagem por categoria administrativa e turno de oferecimento no Estado de Minas Gerais, Belo Horizonte, MG, 2010

Os achados do estudo revelam a ampliação do número de vagas disponibilizadas nos cursos de enfermagem no Estado de Minas Gerais, demonstrando a variação entre instituições pública e privada com tendencia da expansão nesta última, conforme tabela 1.

Tabela 1 - Número de vagas disponibilizadas anualmente nos cursos de graduação em enfermagem por categoria administrativa no Estado de Minas Gerais, Belo Horizonte, MG, 2010

\begin{tabular}{lcc}
\hline Número de vagas anuais & IES Privada & IES Pública \\
\hline Mínimo & 50 & 28 \\
Média & 120,6 & 65,4 \\
Máxima & 600 & 100 \\
\hline
\end{tabular}

Fonte: Brasil - Ministerio da Educação - 2010

Os resultados apresentados na tabela 2 indicam que os cursos de enfermagem no Estado de Minas Gerais apresentam diversidade em relação à carga horária, com variação entre instituições públicas e privadas, sendo que estas tendem a apresentar menor carga horária total nos cursos.

Em relação ao número de semestres para integralização da formação, a variação foi de oito e 10 semestres, com concentração dos cursos com 10 semestres no período noturno.
Tabela 2 - Variação da carga horária dos cursos de graduação em enfermagem por categoria administrativa no Estado de Minas Gerais, Belo Horizonte, MG, 2010

\begin{tabular}{lcc}
\hline Carga horária & IES Privada & IES Pública \\
\hline Mínima & 2800 & 3945 \\
Média & 3783 & 4145 \\
Máxima & 4540 & 4580 \\
\hline
\end{tabular}

Fonte: Brasil - Ministerio da Educação - 2010

\section{DISCUSSÃO}

Os achados permitem afirmar que a distribuição da oferta dos cursos de enfermagem ocorreu em consonância ao desenvolvimento de políticas públicas de educação, atrelado ao modelo político, econômic e-social vigente no país. ${ }^{12}$

No contexto da expansão recente dos cursos de enfermagem, é possível estabelecer uma relação com a Lei de Diretrizes e Bases da Educação Nacional (LDB), promulgada em 1996, como marco que provocou mudanças estruturais no campo da educação superior em geral, com implicações para o ensino de enfermagem.

A promulgação da LDB forneceu autonomia pedagógica, administrativa e de gestão financeira para as instituições de ensino, o que proporcionou um aumento do número de instituições de ensino superior no país ${ }^{13} \mathrm{e}$, consequentemente, no Estado de Minas Gerais. Por outro lado, por sua orientação neoliberal, ${ }^{14}$ essa autonomia fornecida às instituições de ensino, reflexo de uma frágil regulação da educação superior no País, favoreceu o aumento do número de instituições em especial no setor privado, atendendo à lógica mercadológica. Assim, a expansão dos cursos se concentra majoritariamente nas instituições privadas, o que também acompanha as tendências de outros estados brasileiros ${ }^{15} \mathrm{e}$ do cenário nacional. ${ }^{2,16}$

De fato, a privatização do ensino no país é reflexo da política educacional exercida desde a década de 1990 que permitiu o papel do mercado na definição da criação de novos cursos e instituições. Com isso, há a satisfação da demanda por novas vagas proporcionada pelo crescimento da rede privada. ${ }^{13}$ Esse movimento levou também à inversão na proporção de instituições públicas/ privadas que ofereciam o curso de enfermagem, passando a rede privada a ostentar o maior o número de cursos no estado.

O desequilíbrio entre o número de cursos da rede pública e da rede privada expressa a premissa 
mercadológica da educação superior que valoriza o mercado econômico como aspecto fundamental na criação de novos cursos e instituições. ${ }^{2}$

Por outro lado, nota-se um movimento, ainda incipiente, da expansão das instituições públicas, como resposta do investimento das políticas de educação como o Programa de Apoio a Planos de Reestruturação e Expansão das Universidades Federais (REUNI), que induziu no Estado a criação de dois cursos.

Pode-se afirmar, ainda, que a expansão da oferta de cursos de enfermagem contribui para a ampliação do acesso à educação superior no país, não só pelo exponencial aumento do número de cursos, como também pelo aumento de vagas em um mesmo curso; tendência também observada em outros estudos. ${ }^{15}$ Contudo, devemos indagar como será a absorção destes profissionais no mercado de trabalho em saúde.

A análise revela a desigualdade na oferta de cursos entre as macrorregiões do Estado de Minas Gerais e também no interior das regiões. Os cursos estão mais concentrados em regiões com maior desenvolvimento, as quais possuem maior capacidade instalada de recursos humanos qualificados e de infra-estrutura pré-existentes, reafirmando as desigualdades regionais e confirmando a lógica da inserção das instituições em locais potenciais de "consumo". Esse movimento dificulta a formação de profissionais nas regiões do Estado economicamente mais carentes, como as macrorregiões Jequitinhonha, Noroeste e Norte de Minas. Mesmo nestas regiões há uma tendência à concentração dos cursos nos centros urbanos reproduzindo as desigualdades regionais.

Ao mesmo tempo, o investimento de políticas públicas de saúde e educação que incentivem a interiorização e fixação de trabalhadores da saúde, bem como a ampliação do acesso universitário da população dessas regiões parece começar a induzir um movimento de expansão dos cursos nestas regiões, ainda em disparidade com as demais, na tentativa de corrigir a desproporção do quantitativo de profissionais com o número de habitantes. ${ }^{13}$

Outro aspecto que merece análise na oferta dos cursos de graduação é a tendência à flexibilidade, expressa pela variedade de carga horária e número de semestres dos cursos existentes. A flexibilidade, neste caso, pode se tornar um conceito perigoso do ponto de vista da qualidade da formação, em especial quando não se determina que tipo de formação deve ser privilegiada. Com isso, abre-se espaço para que as instituições ofereçam "cursos específicos" com carga horária mínima e que atendam demandas emanadas do mercado, sem um projeto coletivo de formação. ${ }^{17-18}$

\section{CONCLUSÕES}

Os achados do estudo permitem concluir que há uma expansão da oferta dos cursos de enfermagem do Estado de Minas Gerais na qual o setor privado é dominante. A expansão tem relações com as políticas de educação na tentativa de ampliar o acesso à educação superior Visualiza-se que esse contexto, tomado como política de governo, repercute na formação em enfermagem com a expansão vertiginosa do número de cursos e das vagas disponibilizadas em todas as regiões do estado à modelo do que ocorreu no país como um todo.

Os dados permitem afirmar ainda que os cursos de graduação em enfermagem no Estado de Minas Gerais caracterizam-se pela diversidade na organização político-administrativa, turno de oferta e tempo para integralização.

Mesmo diante da incipiência dos dados, aponta-se que as premissas do mercado têm influenciado a criação de escolas de enfermagem.

\section{REFERENNCIAS}

1. Ito EE, Peres AM, Takahashi RT, Leite MMJ. O ensino de enfermagem e as diretrizes curriculares nacionais: utopia x realidade. Rev Esc Enferm USP. 2006 Dez; 40(4):570-5.

2. Teixeira E, Vale EG, Fernandes JD, Sordi MRL. Trajetoria e tendências dos cursos de enfermagem no Brasil. Rev Bras Enferm. 2006 Jul-Ago; 59(4):479-87.

3. Germano RM. O ensino de enfermagem em tempos de mudança. Rev Bras Enferm. 2003 Jul-Ago; 56(4):365-8.

4. Carvalho YM, Ceccim RB. Formação e educação em saúde: aprendizados com a saúde coletiva. In: Campos GWS, organizador. Tratado de saúde coletiva. São Paulo (SP): Hucitec; 2006. p.149-82

5. Brasil. Ministério da Educação e Cultura. Portaria n. 1721 de 16 de dezembro de 1994. Dispõe sobre o Currículo Mínimo do Curso de Enfermagem. Diário Oficial da União, Brasília, 16 Dez 1994. Seção 1:19301-2.

6. Sena RR, Egry EY. A enfermagem nos Projetos UNI: contribuição para um novo projeto político para a enfermagem brasileira. São Paulo (SP): Hucitec; 1998.

7. Brasil. Ministério da Educação e Cultura. Lei 9394 de 20 de dezembro de 1996. Estabelece as Diretrizes e bases da Educação Nacional. Diário Oficial da União, Brasília, 23 Dez 1996. Seção 1:27833-41. 
8. Silva KL. Movimento de mudança na educação de enfermagem: construindo a integralidade do cuidado na saúde [dissertação]. Belo Horizonte (MG): UFMG/ Programa de Pós-Graduação em Enfermagem; 2005.

9. Silva KL, Sena RR. Integralidade do cuidado na saúde: indicações a partir da formação do enfermeiro. Rev Esc Enferm USP. 2008 Mar; 42(1):48-56.

10. Fernandes JD. Diretrizes curriculares e projetos pedagógicos de cursos de graduação em enfermagem. In: Teixeira E, organizadora. O ensino de graduação em enfermagem no Brasil: o ontem, o hoje e o amanhã. Brasília (DF): INEP, 2006. p.41-54.

11. Sena RR, Seixas CT, Silva KL. Practices in community health toward equity: contributions of brazilian nursing. ANS Adv Nurs Sci. 2007 Out-Dez; 30(4):343-52.

12. Silva MG, Fernandes JD, Teixeira GAS, Silva RMO. Processo de formação da(o) enfermeira(o) na contemporaneidade: desafios e perspectivas. Texto Contexto Enferm. 2010 Jan-Mar; 19(1):176-84.

13. Vieira ALS, Garcia ACP, Filho AA, Pierantoni CR, Ferraz CP, Oliveira ES, et al. Tendências do sistema educativo no Brasil: medicina, enfermagem e odontología. In: Ministério da Saúde. Observatório de recursos humanos em saúde no Brasil: estudos e análises. Brasília (DF): MS; 2004.

14. Xavier ML, Baptista SS. Associação Brasileira de Enfermagem no contexto da reforma educacional de 1996. Rev Bras Enferm. 2010 Mar-Abr; 63(2):257-63.

15. Rodrigues RM, Caldeira S. Formação na graduação em enfermagem no estado do Paraná. Rev Bras Enferm. 2009 Maio-Jun; 62(3):417-23.

16. Varella TC, Pierantoni CR. A migração de enfermeiros: um problema de saúde pública. Rev Bras Saude Matern Infant. 2007 Abr-Jun; 7(2):199211.

17. Silva KL, Sena RR. Nursing education: seeking critical-reflexive education and professional competencies. Rev Latino-am Enfermagem. 2006 Set-Out; 14(5):755-61.

18. Bagnato MHS, Rodrigues RM. Diretrizes curriculares da graduação de enfermagem: pensando contextos, mudanças e perspectivas. Rev Bras Enferm. 2007 Set-Out; 60(5):507-12. 